\title{
Short-range correlation in an electron gas: A scattering approach
}

\author{
I. Nagy, ${ }^{1,2}$ J. I. Juaristi, ${ }^{3,4}$ R. Díez Muiño, ${ }^{2}$ and P. M. Echenique ${ }^{2,3,4}$ \\ ${ }^{1}$ Department of Theoretical Physics, Institute of Physics, Technical University of Budapest, H-1521 Budapest, Hungary \\ ${ }^{2}$ Donostia International Physics Center DIPC, 20080 San Sebastián, Spain \\ ${ }^{3}$ Departamento de Física de Materiales, Facultad de Ciencias Químicas, Universidad del País Vasco, Aptdo. 1072, \\ 20080 San Sebastián, Spain \\ ${ }^{4}$ Centro Mixto CSIC-UPV/EHU, Aptdo. 1072, 20080 San Sebastián, Spain
}

(Received 2 October 2002; published 6 February 2003)

\begin{abstract}
The pair-correlation function at zero interparticle separation $g(0)$ of an interacting electron gas is derived by an averaging procedure using the exact enhancement factor for scattered waves of electrons in a model potential. The range of the screened potential is fixed by a physically motivated constraint. Agreement with the result of a many-body method based on summation of ladder diagrams for electron-electron interactions is established. Possible applications of the potential are discussed as well. A nontrivial density-scaling in the thermal resistivity of metals is predicted.
\end{abstract}

DOI: 10.1103/PhysRevB.67.073102

PACS number(s): 71.10.Ca, 71.45.Gm

The pair-distribution function $g(r)$ of a homogeneous electron gas describes a relative behavior of pairs of spinhalf fermions. It is defined as the probability $[g(r) \geqslant 0]$ that another particle is at $r$ if there is already one at $r=0$. A well-motivated $g(r)$ for the uniform system is, in addition to its intrinsic theoretical interest, the starting point for modeling averaged exchange-correlation hole of a many-electron system of nonuniform density.

For an unpolarized gas $g(r)=(1 / 2)\left[g_{\uparrow \uparrow}(r)+g_{\uparrow \downarrow}(r)\right]$, in which $g_{\uparrow \uparrow}(r)$ describes both exchange and Coulomb correlations among the equal-spin electrons, while $g_{\uparrow \downarrow}(r)$ describes Coulomb correlation among electrons with opposite spin. For a system of noninteracting electrons described by a single Slater determinant, there are no correlation effects, only exchange. This latter is due solely to the Pauli exclusion principle. Therefore, in the Hartree-Fock (HF) approximation $g(r=0)=(1 / 2)$, because $g_{\uparrow \uparrow}(r=0)=0$ and $g_{\uparrow \downarrow}(r)=1$.

A lower energy state of the interacting electron gas than the HF one is governed by correlated motions of electron pairs. The value of $g\left(r=0, r_{s}\right)$, at a given density $n_{0}$ $=3 /\left(4 \pi r_{s}^{3}\right)$ of the system, reflects most clearly the effect of Coulomb interactions, since $g_{\uparrow \uparrow}\left(0, r_{s}\right)=0$ by the Pauli constraint. A standard mean-field (Hartree) method, based on the charge-polarization concept and the random-phase approximation (RPA) for the dielectric function, gives a negative value for $g\left(0, r_{s}\right)$ already at $r_{s} \geqslant 0.8$, as was shown and analyzed by Hedin. ${ }^{1}$ This method violates the requirement of the exclusion principle and, furthermore, treats the short-range correlation due to the Coulomb force between electrons of antiparallel spin perturbatively. The RPA is a method to account the long-range part of the pair-distribution function, but fails to distinguish between electrons of equal and opposite spin. $^{2}$

The considerations of interactions, using powerful manybody methods, provide the exact $(e)$ asymptotic $^{3}\left(r_{s} \rightarrow 0\right)$, and the well-established ${ }^{4}$ electron-electron ladder $(l)$ results for the average $g\left(0, r_{s}\right)$ functions

$$
2 g^{e}\left(0, r_{s} \rightarrow 0\right)=1-\beta_{e} r_{s},
$$

where $\beta_{e}=(2 \alpha / 5 \pi)\left(\pi^{2}+6 \ln 2-3\right)$ with $\alpha=(4 / 9 \pi)^{1 / 3}$ thus $\beta_{e} \cong 0.732$, and

$$
2 g^{l}\left(0, r_{s}\right)=\left[\sum_{m=0}^{\infty} \frac{u^{2 m}}{m !(m+1) !}\right]^{-2}=\left[\frac{u}{I_{1}(2 u)}\right]^{2}
$$

in which $u^{2}=4 \alpha r_{s} / \pi$ and $I_{1}(x)$ is the first-order modified Bessel function. From Eq. (2) one obtains $2 g^{l}\left(0, r_{s} \rightarrow 0\right)=1$ $-\beta_{l} r_{s}$ with $\beta_{l}=4 \alpha / \pi \cong 0.663$.

A recent theoretical effort, using an effective two-particle interaction with no empirical parameter to account for twobody correlations, gave surprisingly accurate numerical values for $g\left(0, r_{s}\right)$ even for the mentioned high-density limit. ${ }^{5}$ This effort was based on the scattering interpretation and implementation of Overhauser. ${ }^{6}$ Note at this important point, that the concept of effective, state-dependent pair potentials has been successfully applied in the description of reduced Coulomb repulsions between antiparallel-spin electrons of the main shells of atoms. ${ }^{7}$

Overhauser used a physically motivated, the so-called point charge in the continuum or Wigner-Seitz model of screening, finite-range potential of the form ${ }^{6,8,9}$

$$
V_{\mathrm{WS}}(r)=\frac{1}{r}\left[1-\frac{r}{2 r_{s}}\left(3-\frac{r^{2}}{r_{s}^{2}}\right)\right],
$$

with $V_{\mathrm{WS}}(r)=0$ for $r \geqslant r_{s}$, and obtained a first-iteration result from the two-particle scattering Schrödinger equation

$$
2 g^{\circ}\left(0, r_{s}\right)=\frac{64}{\left(8+3 r_{s}\right)^{2}} .
$$

Using this potential (with additional probabilistic arguments on its capability) and performing a complete partial-wave expansion at a fixed relative scattering $(k)$ momentum, introducing ${ }^{5}$ the proper weighting over this momentum by its normalized probability distribution function 


$$
P(k)=24 \frac{k^{2}}{k_{F}^{3}}-36 \frac{k^{3}}{k_{F}^{4}}+12 \frac{k^{5}}{k_{F}^{6}},
$$

where $k \in\left[0, k_{F}\right]$ and $k_{F}^{3}=3 \pi^{2} n_{0}$, Gori-Giorgi and Perdew obtained $^{5}$ a remarkably similar numerical result for $g\left(0, r_{s}\right)$, in the range of $r_{s} \in[0,10]$, to the one prescribed by Eq. (2).

The established and surprising agreement may generate, in our opinion, quite natural questions. Is the agreement due to the screened form of the applied potential? If the screening is so important, how important is the particular finite-range version? The present paper is devoted to a detailed theoretical investigation on these questions, and provides another form for the screened potential resulting in an almost complete agreement with $g^{l}\left(0, r_{s}\right)$ for the $r_{s} \in[0,10]$ range of the density parameter, too.

Generally, for a given potential one can calculate the socalled enhancement factor defined by the scattering-wave $\left(\Psi_{k}\right)$ solution of the Schrödinger equation as

$$
E(k)=\left|\frac{\Psi_{k}(0)}{\Psi_{k}^{0}(0)}\right|^{2},
$$

where $\Psi_{k}^{0}$ is the field-free solution. Thus, using Eq. (5) one obtains

$$
2 g\left(0, r_{s}\right)=\int_{0}^{k_{F}} d k P(k) E(k)
$$

For a repulsive (unscreened) Coulomb interaction between equal-mass and unit-charge particles $E_{c}(k)=(\pi / k)$ $\times[\exp (\pi / k)-1]^{-1}$, thus for the $r_{s} \rightarrow 0$ limit the result is

$$
2 g^{c}\left(0, r_{s} \rightarrow 0\right)=1-\beta_{c} r_{s},
$$

in which $\beta_{c}=6 \pi \alpha / 5 \cong 1.964$. The asymptotic result of Eq. (8) heralds that the mathematical tuning of the coefficient in the $r_{s}$ term requires physical screening in an effective twobody model. The required $k_{F}$ dependence of the screening length is investigated now by using an auxiliary example.

Let us introduce a fixed impurity potential of Yukawa-type $V_{Y}(r)=(1 / r) \exp (-\lambda r)$ into the electron gas and calculate the induced charge-density $\Delta n(r)$ using standard ${ }^{10}$ perturbation theory based on plane waves. The result for $\Delta n(0)$ is as follows:

$$
\Delta n(0)=\frac{2 k_{F}^{2}}{3 \pi^{2}} x F\left(\frac{1}{2}, \frac{3}{2} ; \frac{5}{2} ; x^{2}\right),
$$

where $x=2 k_{F} /\left(\lambda^{2}+4 k_{F}^{2}\right)^{1 / 2}$ and $F$ is the hypergeometric function. Defining a pair-correlation function as $G\left(0, r_{s}\right)=1$ $-\Delta n(0) / n_{0}$ for the auxiliary model, one can see that only a $\lambda \sim k_{F}$ scaling could allow tuning of $\Delta n\left(0, r_{s}\right)$ without violating the $G\left(0, r_{s} \rightarrow 0\right)=1-\beta r_{s}$ character in the relevant high-density limit. For any other $\lambda \sim k_{F}^{m}(0<m<1)$ scaling the limit value at $r_{s} \rightarrow 0$ remains unchanged; one gets, in these cases, always the corresponding (static fixed impurity) Coulombic limit with $\beta=3 \pi \alpha / 2 \cong 2$.455. In addition, we note that with the $\lambda_{\mathrm{TF}}=\left(4 k_{F} / \pi\right)^{1 / 2}$ Thomas-Fermi value in Eq. (9) one has $G\left(0, r_{s}\right)=0$ at about $r_{s}=0.8$ already.

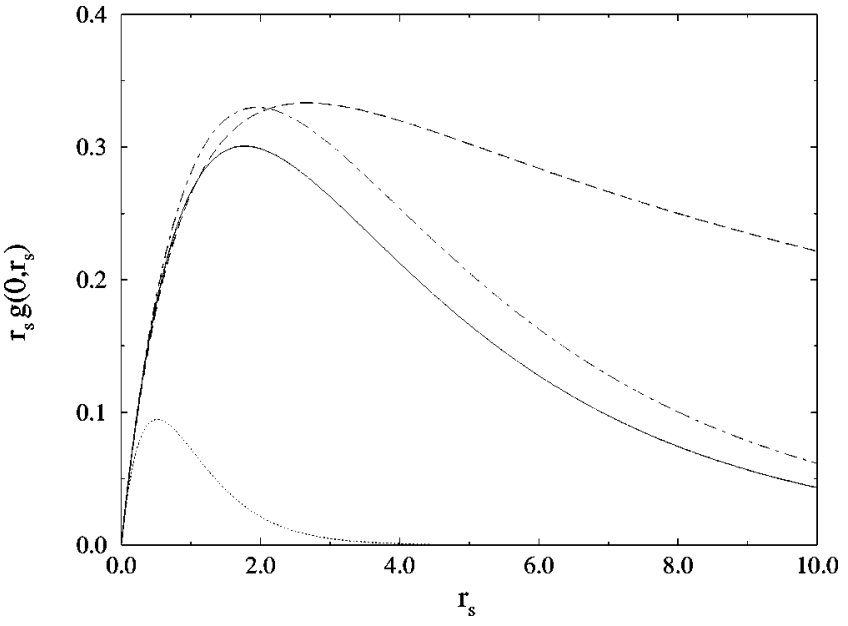

FIG. 1. Pair-correlation functions, plotted as $r_{s} g\left(0, r_{s}\right)$, at zero interparticle separation as a function of $r_{s}$. The solid and dashdotted curves are the results of the present work. The dotted curve is based on the same averaging procedure but rests on the Coulombic enhancement factor $E_{c}(k)$. The dashed curve refers to Eq. (4). See the text for further details.

Supported by the above detailed investigations, we describe the screened potential in our treatment on the paircorrelation problem by a Hulthen-type ${ }^{11,12}$ form

$$
V_{H}(r)=\frac{\Lambda}{e^{\Lambda r}-1} .
$$

This form allows ${ }^{13}$ an exact representation of the corresponding enhancement factor needed to Eq. (7) as

$$
E_{H}(k)=\frac{\pi}{k} \frac{\sinh (v)}{\cosh (w)-\cosh (v)},
$$

where $v=(2 \pi k / \Lambda)$ and $w=v\left(1+\Lambda / k^{2}\right)^{1 / 2}$. Furthermore, motivated by the success 5 of the model proposed by Overhauser and, especially, his iteration method for the $s$ wave [the radial wave-function behaves as $R(r) \sim(1 / r)$ ], we use the

$$
\int_{0}^{r_{S}} d r r^{2} \frac{1}{r} V_{W S}(r)=\int_{0}^{\infty} d r r^{2} \frac{1}{r} V_{H}(r),
$$

constraint and obtain $\Lambda=(2 \pi / 3)^{2} / r_{s} \cong 2.285 k_{F}$. Note that in standard scattering theory ${ }^{14}$ the above-defined weighted average of a potential is related, for the attractive case, to the convergence of the Born-series and appearance of a bound state.

Using this prescription for $\Lambda$ in Eq. (11) for $E_{H}(k, \Lambda)$, the remaining single integral in Eq. (7) with Eq. (5) is performed numerically. In Fig. 1 we compare the present $g^{H}\left(0, r_{s}\right)$ result (solid curve) with other estimates of $g\left(0, r_{s}\right)$. The dashed curve refers to Eq. (4) obtained, in a first-iteration analytical method, by Overhauser. The dotted curve is based on the Coulomb enhancement factor $E_{c}(k)$. The dash-dotted curve corresponds to the present procedure, but with $\Lambda_{s}^{2}$ $=20 \zeta(3) / r_{s}^{2}$ for screening (see, below), where the Riemannzeta function is $\zeta(3) \cong 1.2$. The result of the many-body 
method (summing the ladder diagrams for electron-electron interactions) for $g^{l}\left(0, r_{s}\right)$ is not plotted: the $g^{l}\left(0, r_{s}\right)$ and $g^{H}\left(0, r_{s}\right)$ [with $\Lambda$ based on Eq. (12)] functions practically coincide in the $r_{s} \in[0,10]$ range. The deviation between them remains very moderate even at $r_{s}=20$. At this density parameter $r_{s} g^{l}\left(0, r_{s}\right) \cong 0.0032$ while $r_{s} g^{H}\left(0, r_{s}\right) \cong 0.0035$. The mathematical limits for $r_{s} \rightarrow \infty$ are, however, different. By analytical expansions in Eqs. (2) and (11) we obtain

$$
\begin{gathered}
r_{s} g^{l}\left(0, r_{s} \rightarrow \infty\right)=r_{s} 2 \pi u^{3} e^{-4 u} \cong 3.4 r_{s}^{5 / 2} e^{-3.26 \sqrt{r_{s}}}, \\
r_{s} g^{H}\left(0, r_{s} \rightarrow \infty\right) \cong 2 \pi r_{s}^{2} e^{-3 \sqrt{r_{s}} .}
\end{gathered}
$$

One can conclude, that it is the special dependence of the screening length on $k_{F}$, and not the finite- or longer-range form of a two-body effective potential with no empirical parameter, that determines the good performance of $g\left(0, r_{s}\right)$ obtained by Gori-Giorgi and Perdew and in the present work for the interesting range of $r_{s}$. The useful constraint given by Eq. (12) may provide a background to restrict other, i.e., $V_{Y}(r)$, one-parametric potential forms with Coulomb singularity. Proper treatments, such as a ladder or a two-body exact scattering, of the singularity satisfy the electron-electron cusp condition. This fact, and the effective potential-range put rather stringent conditions on the possible $g\left(0, r_{s}\right)$ values.

We have investigated another (related to the nonlinearity question) aspect of scattering by using the potentials $V_{\mathrm{WS}}(r)$ and $V_{H}(r)$. The Hulthen-type potential is fixed with $\Lambda$ of Eq. (12) and the $\Lambda_{s}^{2}=20 \zeta(3) / r_{s}^{2}$ value. This $\Lambda_{s}$ value is obtained by a forward-limit constraint, i.e., via the Fourier-transforms of $V_{\mathrm{WS}}(r)$ and $V_{H}(r)$ at $q=0$ as

$$
\int_{0}^{r_{s}} d r r^{2} V_{\mathrm{WS}}(r)=\int_{0}^{\infty} d r r^{2} V_{H}(r) .
$$

The leading $(l=0)$ phase shifts, $\delta_{0}(k)$, calculated at the maximal relative momentum $k=k_{F}$ from the Schrödinger equation, ${ }^{15}$ are exhibited in Fig. 2 for the metallic range $r_{s}$ $\in[1,6]$. Solid and dash-dotted curves are based on the $\Lambda$ and $\Lambda_{s}$ values in $V_{H}(r)$, respectively, while the dotted curve on the $V_{\mathrm{WS}}(r)$ potential. The obtained phase shifts are not small showing that a nonpertubative method is required to characterize them. Furthermore, a comparison of curves heralds that the $g\left(0, r_{s}\right)$ (Fig. 1) and $\delta_{0}\left(k_{F}\right)$ quantities sample in a different way the strength of the two-body interaction.

The established success based on an effective potential to model correlated motions of antiparallel-spin electrons and obtain a ground-state characteristic, $g\left(0, r_{s}\right)$, of an interacting electron gas may raise a natural question: Is this potential an acceptable one to the theoretical description of physical quantities usually considered as determined by particleparticle (in different relative spin states) scattering at the Fermi level? The scattering lifetime (decay) is recently a topic of great interest. ${ }^{16}$ Similarly, a scattering rate is a key quantity in various low-temperature transport properties. ${ }^{17}$ The mentioned lifetime is, mainly, determined by the smallmomentum transfer $(q \rightarrow 0)$ limit of an effective interaction, while this work addressed the short-range $(q \rightarrow \infty)$ limit. As

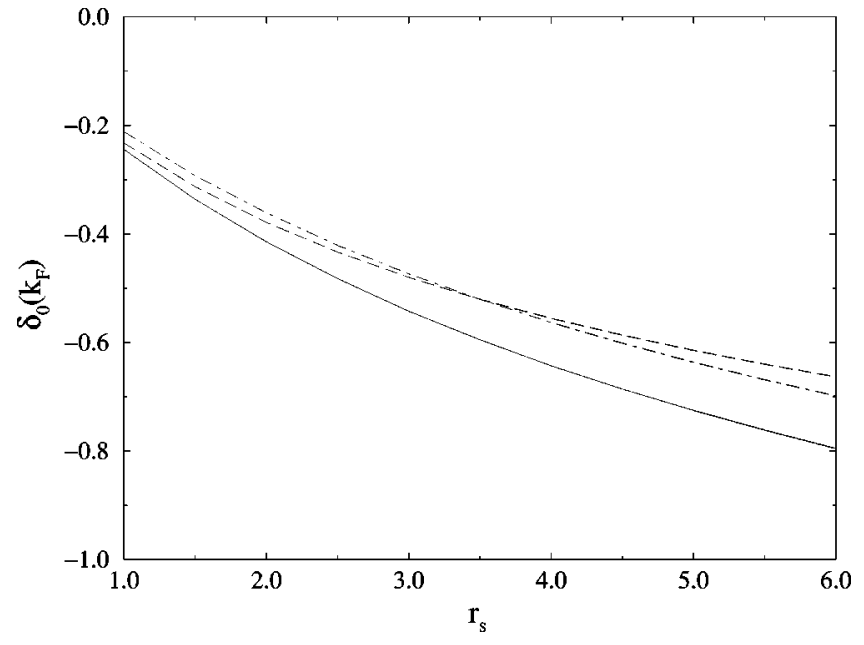

FIG. 2. The leading phase shifts $\left[\delta_{0}\left(k_{F}\right)\right]$ for the metallic range: $r_{s} \in[1,6]$. The solid and dash-dotted curves are based on Eq. (10) with Eqs. (12) and (15), respectively, while the dashed one is obtained by Eq. (3). The Schrödinger equation with reduced mass $\mu$ $=(1 / 2)$ for electron-electron scattering is used in all cases.

we already noted, the $q \rightarrow 0$ limit is properly treated by the RPA. On the other hand, scattering rates in the conduction characteristics contain weighting-out factors for the forward direction and, therefore, may be more sensitive to the shortrange part of the two-body interaction. Reasonable description of the interparticle interaction at close range is a prerequisite in the proper treatment of the so-called $\mathrm{GW}$ approximation, too. ${ }^{18}$

The special sensitivity with which the thermal resistivity $W_{e e}\left(T, r_{s}\right)$, at a given temperature $T$, and the scattering lifetime depend on the screening length $\lambda_{\mathrm{sc}}^{-1}\left(r_{s}\right)$ of oneparametric effective interactions was already clearly stated. ${ }^{19,20}$ Using the standard $W_{e e} \sim \operatorname{Tr}_{s}^{7 / 2} / \lambda_{\text {sc }}^{3}\left(r_{s}\right)$ expression, ${ }^{19}$ here we argue that the experimentally ${ }^{21,22}$ verified very sharp increase of $W_{e e}\left(r_{s}\right)$ by growing $r_{s}$ of metals maybe, at least partly, due to the form of $\lambda_{\mathrm{sc}}\left(r_{s}\right)$ in the antiparallel-spin scattering channel. In an average mean-field description $\lambda_{\mathrm{sc}} \sim \lambda_{\mathrm{TF}} \sim 1 / r_{s}^{1 / 2}$ for all-kind of scattering, while the present study suggests a $\lambda_{\mathrm{sc}} \sim \Lambda \sim 1 / r_{s}$ scaling, thus a $W_{e e}^{\uparrow \downarrow} \sim \operatorname{Tr}_{s}^{13 / 2}$ channel-character instead of the $W_{e e} \sim \operatorname{Tr}_{s}^{10 / 2}$ mean-field-based behavior.

We quantify our statement by a representative example which covers a broad-range of the density via the $r_{s}$ parameters. Experimental results on $W_{e e}$ obtained for $R b\left(r_{s}\right.$ $=5.2)$ target by $\mathrm{Cook}^{21}$ and for $\mathrm{Cu}\left(r_{s}=2.66\right)$ target by Laubitz $^{22}$ give, using the mean-value data, the $R=350 / 4$ $\cong 87$ ratio for $W_{e e}(R b) / W_{e e}(C u)$. Our scaling results in $R$ $=(5.2 / 2.66)^{13 / 2} \cong 80$, while the usual one in $R=(5.2 / 2.66)^{5}$ $\cong 28$. The nontrivial scaling, and the important role of the antiparallel-spin scattering channel behind of it, may contribute to the understanding of the abovementioned sharp (see Fig. 1 of Ref. 19) increase of $W_{e e}\left(r_{s}\right)$ of metals with $r_{s}$ $\in[2.6,5.6]$.

Finally, by returning to the basic problem of this paper, a similar analysis as the present one for the $3 \mathrm{D}$ electron gas 
would be desirable for the 2D fermion system, where the forward-backward limits of effective interactions are equally important in lifetime calculations. ${ }^{23}$ Certain scattering aspects, using a Wigner-Seitz-like potential, ${ }^{9}$ were already investigated $^{24}$ for the important two-dimensional model. The so-called double-photoelectron emission, as a tool ${ }^{25}$ for correlation imaging, could give further informations on the mutual interaction between electrons.
The work of I.N. was supported by OTKA (Grant Nos. T034363 and T038162). J.I.J., R.D.M., and P.M.E. acknowledge support from UPV/EHU and Eusko Jaurlaritza, Ministerio de Educación y Cultura (Grant No. BFM 2001-0076), and Iberdrola S.A. R.D.M. acknowledges support by the Gipuzkoako Foru Aldundia. I.N. is thankful to N.H. March for enlightening discussions and $\mathrm{H}$. Yasuhara for the kind help.
${ }^{1}$ L. Hedin, Phys. Rev. 139, A796 (1965).

${ }^{2}$ P. Fulde, Electron Correlation in Molecules and Solids (SpringerVerlag, Berlin, 1991), Sec. 10.2.

${ }^{3}$ D. J. W. Geldart, Can. J. Phys. 45, 3139 (1967); J. C. Kimball, Phys. Rev. B 14, 2371 (1976).

${ }^{4}$ H. Yasuhara, Solid State Commun. 11, 1481 (1972); J. Phys. Soc. Jpn. 36, 361 (1974).

${ }^{5}$ P. Gori-Giorgi and J. P. Perdew, Phys. Rev. B 64, 155102 (2001).

${ }^{6}$ A. W. Overhauser, Can. J. Phys. 73, 683 (1995).

${ }^{7}$ J. A. Alonso and N. A. Cordero, J. Phys. B 29, 1629 (1996).

${ }^{8}$ M. Seidl, J. P. Perdew, and M. Levy, Phys. Rev. A 59, 51 (1999).

${ }^{9}$ I. Nagy, Phys. Rev. B 60, 4404 (1999).

${ }^{10}$ W. Jones and N. H. March, Theoretical Solid State Physics (Wiley, New York, 1973), Eq. (2.3.11).

${ }^{11}$ L. Hulthen, Arkiv. Mat. Fys. Astr. 28A(5), 1 (1942).

${ }^{12}$ J. Lindhard and A. Winter, Nucl. Phys. A 166, 413 (1971).

${ }^{13}$ P. F. Meier, Helv. Phys. Acta 48, 227 (1975).

${ }^{14}$ N. F. Mott and H. S. W. Massey, The Theory of Atomic Collisions (Oxford University Press, Oxford, 1965), Sec. V.3.
${ }^{15}$ F. Calogero, Variable Phase Approach to Potential Scattering (Academic Press, New York, 1967).

${ }^{16}$ P. M. Echenique, J. M. Pitarke, E. V. Chulkov, and A. Rubio, Chem. Phys. 251, 1 (2000).

${ }^{17}$ G. D. Mahan, Many-Particle Physics (Plenum Press, New York, 1981), Sec. 10.4.

${ }^{18}$ B. Holm and U. von Barth, Phys. Rev. B 57, 2108 (1998).

${ }^{19}$ N. Iwamoto, Phys. Rev. B 59, 9687 (1999), and references therein.

${ }^{20}$ I. Nagy, J. I. Juaristi, and P. M. Echenique, Phys. Rev. B 63, 035102 (2000).

${ }^{21}$ J. G. Cook, Can. J. Phys. 56, 871 (1979).

${ }^{22}$ M. J. Laubitz, Phys. Rev. B 2, 2252 (1970).

${ }^{23}$ J. Jungwirth and A. H. MacDonald, Phys. Rev. B 53, 7403 (1996).

${ }^{24}$ M. Polini, G. Sica, B. Davoudi, and M. P. Tosi, J. Phys.: Condens. Matter 13, 3591 (2001).

${ }^{25}$ N. Fominykh, J. Berakdar, J. Henk, and P. Bruno, Phys. Rev. Lett. 89, 086402 (2002). 